This manuscript has been accepted for publication in Cognition.

Preprint first posted online June 25, 2018.

It is made available under a CC-By Attribution 4.0 International license. The most current version of the preprint can be found at https://dx.doi.org/10.17605/OSF.IO/VRBGH

\title{
Probabilistic rejection templates in visual working memory
}

\author{
Andrey Chetverikovva,b, Gianluca Campana ${ }^{\mathrm{d}, \mathrm{e}}$, \& Árni Kristjansson ${ }^{\mathrm{b}, \mathrm{f}}$ \\ a - Center for Cognitive Neuroimaging, Donders Institute for Brain, Cognition and Behavior, \\ Radboud University; ${ }^{\mathrm{b}}$ - Laboratory for Visual Perception and Visuomotor Control, Faculty of \\ Psychology, School of Health Sciences, University of Iceland, Reykjavik, Iceland; ${ }^{\mathrm{c}}$ - Cognitive \\ Research Lab, Russian Academy of National Economy and Public Administration, Moscow, \\ Russia; ${ }^{\mathrm{d}}$ - Department of General Psychology, University of Padova, Padova, Italy; e - Human \\ Inspired Technology Research Centre, University of Padova, Italy; ${ }^{\mathrm{f}}$ - School of Psychology, \\ National Research University Higher School of Economics.
}

Author Note

Correspondence should be addressed to: Andrey Chetverikov, Donders Institute for Brain, Cognition and Behavior, Center for Cognitive Neuroimaging, Kapittelweg 29, 6525 EN Nijmegen, The Netherlands. Email: a.chetverikov@donders.ru.nl. The preprint of this paper is available at https://dx.doi.org/10.17605/OSF.IO/VRBGH The data from the experiments reported in this paper and scripts for simulations and analyses are available at https://osf.io/rg2h8 Word count: 4740. 
Abstract

2 Our interactions with the visual world are guided by attention and visual working memory.

3 Things that we look for and those we ignore are stored as templates that reflect our goals and the

4 tasks at hand. The nature of such templates has been widely debated. A recent proposal is that

5 these templates can be thought of as probabilistic representations of task-relevant features.

6 Crucially, such probabilistic templates should accurately reflect feature probabilities in the

7 environment. Here we ask whether observers can quickly form a correct internal model of a

8 complex (bimodal) distribution of distractor features. We assessed observers' representations by

9 measuring the slowing of visual search when target features unexpectedly match a distractor

10 template. Distractor stimuli were heterogeneous, randomly drawn on each trial from a bimodal

11 probability distribution. Using two targets on each trial, we tested whether observers encode the

12 full distribution, only one peak of it, or the average of the two peaks. Search was slower when

13 the two targets corresponded to the two modes of a previous distractor distribution than when

14 one target was at one of the modes and another between them or outside the distribution range.

15 Furthermore, targets on the modes were reported later than targets between the modes that, in

16 turn, were reported later than targets outside this range. This shows that observers use a correct

17 internal model, representing both distribution modes using templates based on the full

18 probability distribution rather than just one peak or simple summary statistics. The findings

19 further confirm that performance in odd-one out search with repeated distractors cannot be

20 described by a simple decision rule. Our findings indicate that probabilistic visual working

21 memory templates guiding attention, dynamically adapt to task requirements, accurately

22 reflecting the probabilistic nature of the input.

23 Keywords: attentional templates, visual working memory, probabilistic representations, visual 24 ensembles, summary statistics, visual search. 


\section{Probabilistic rejection templates in visual working memory}

27 Our senses are constantly bombarded with an overwhelming amount of information that needs to be filtered by the brain to guide action. This information, however, is not completely chaotic. For example, leaves on a tree usually have similar colors, and colors within a single leaf would be more similar to each other than to another leaf. Probabilistic models of vision (Bejjanki, Beck, Lu, \& Pouget, 2011; Feldman, 2014; Girshick, Landy, \& Simoncelli, 2011; Kersten, Mamassian, \& Yuille, 2004; Ma, 2012; Rao, Olshausen, \& Lewicki, 2002) suggest that the brain utilizes existing correlations in the environment and uses them in perception. However, some of the incoming information is not relevant for current behavior, and it is important to reject it while processing other stimuli in more detail. Traditionally, the rejection of irrelevant information within a specific feature dimension (e.g., orientation) is thought to be based on specific feature values (Woodman, Carlisle, \& Reinhart, 2013). Here we ask whether such rejection can instead be based on probabilistic templates and whether such templates accurately reflect the probabilities of distractor features. If this is the case, then probabilistic inference in the brain does not start with perception, but sooner, when to-be-rejected templates are formed (based on previously encountered stimuli) to optimize the prioritization of what is perceived.

Imagine a radiologist looking for signs of tumor in X-ray scans. Malignant signs can take many forms so the targets to look for are diverse. By many accounts, search in this and other contexts is thought to be guided by templates held in visual working memory (Woodman et al., 2013). These templates reflect what one should look for, but may also reflect what should be ignored (Arita, Carlisle, \& Woodman, 2012; Won \& Geng, 2018). For example, distractors such as the rib cage on a lung scan are salient but not informative and radiologists can therefore ignore them. It is well known that information about to-be-ignored stimuli or features is kept in memory, but the way they are represented is still unknown.

There are capacity limits in the amount of information that can be stored in visual working memory templates (Bundesen, 1990; Grubert \& Eimer, 2013; Vickery, King, \& Jiang, 2005), with some authors even suggesting that only one template containing a single feature value can guide attention at any given time (Oberauer, 2002; Olivers, Peters, Houtkamp, \& Roelfsema, 2011; van Moorselaar, Theeuwes, \& Olivers, 2014). Alternatively, templates could

55 be conceptualized as probabilistic entities of varying precision (Bays, 2015) rather than matches to exact feature values. While previous studies found some support for this, observers typically 
57 reported features of single items (Ma, Husain, \& Bays, 2014). However, in the real world such 58 isolated features practically never occur. Furthermore, with a few exceptions (Arita et al., 2012;

59 Won \& Geng, 2018), templates for ignored information are rarely studied. For any inference

60 based on the probabilistic representations, it is crucial that the internal model used by observers

61 accurately reflects the environment. Here, we provide strong evidence for the probabilistic

62 template view by showing that visual working memory templates for rejection mirror the

63 probability distribution of distractor features.

64 Our observers searched for two oddly oriented targets among distractors randomly drawn

65 from a bimodal orientation distribution. To expose observers' templates, after a sequence of

66 learning trials with distractors randomly drawn from a bimodal distribution, targets on test trials

67 could either correspond to regions of feature space previously used for distractors, fall in

68 between the modes of the bimodal distribution, or have feature values outside the previous

69 distribution range. We assume that observers' templates reflect what has been relevant on recent

70 trials. If templates contain features of distractors to be ignored, which then become targets on test

71 trials, search should be slower than otherwise (Chetverikov, Campana, \& Kristjánsson, 2016;

72 Kristjánsson \& Driver, 2008; Lamy, Antebi, Aviani, \& Carmel, 2008; Maljkovic \& Nakayama,

73 1994; Wang, Kristjánsson, \& Nakayama, 2005). Crucially, experiments with varied set size and

74 trial numbers show that learning in this paradigm cannot be explained by the sampling of a few

75 items (Chetverikov, Campana, \& Kristjánsson, 2017d, 2017b). It also cannot be explained by

76 simple decision rule learning (e.g., all stimuli that have features in a certain range are

77 distractors), because observers response times, on average, reflect the shape of the distractor

78 distribution rather than just a boundary between a target and distractors (Chetverikov et al., 2016,

79 2017b; Chetverikov, Campana, \& Kristjánsson, 2017c; Chetverikov, Hansmann-Roth, Tanrikulu,

80 \& Kristjansson, 2019). However, it is not yet clear whether each single set of learning trials can

81 feed observers' templates with the feature probability distribution of distractors, nor is it clear

82 how accurately the information is stored in the templates.

83 Under the strong probabilistic template hypothesis, templates would include information

84 about both peaks of a bimodal distribution. That is, observers would develop an accurate internal

85 model for the task and the template would accurately reflect the information about the full

86 probability distribution. Alternatively, templates might include only a single peak (e.g., the

87 attended one), or might reflect only the summary statistics, such as the averages of the whole 
88 distribution (Alvarez, 2011). Using a two-target search task we were able to test whether observers encode both peaks of a distribution following a single learning sequence. The

90 predictions of these models (see Simulations) are qualitatively different regarding both the order

91 in which targets are reported in a two-target search, and search times. If observers accurately

92 encode a bimodal distribution, on trials with a target on a peak and target between peaks, targets

93 between the peaks (associated with a lower distractor probability) should be reported before

94 targets on peaks (associated with the highest distractor probability, Figure 1A). In contrast, if

95 only one peak is encoded or if the whole distribution is averaged, targets on peaks would be

96 associated with a lower distractor probability and should be reported no later than targets

97 between the peaks (associated with lower distractor probability in this case). Notably, while all

98 three hypotheses postulate that observers can use probabilistic inference, only the first one

99 assumes that the distractor probability distribution is encoded accurately, that is, that the

100 observers use relatively accurate probabilistic templates.

A

Physical distribution
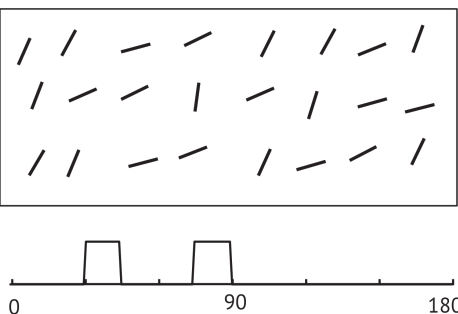

Single template

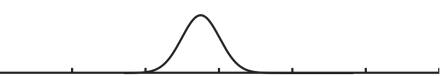

Averaged template

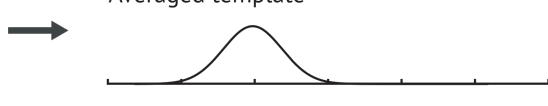

Probabilistic bimodal template

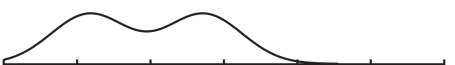

B Learning trials

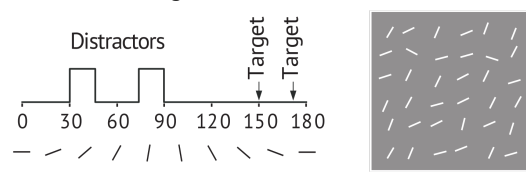

Test trials
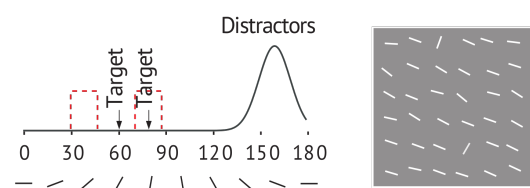

Figure 1. Panel A: The same physical bimodal distribution can be represented in different ways. Panel B:

103 Example learning and test trials with distractor distributions and targets shown on the left. 


\section{Experiment}

106

107

Ethics Statement. The study was approved by the ethics committee of St. Petersburg State University (\#75, 21.06.2017). All participants signed a consent form before taking part in the study.

Participants. Fifteen observers (ten female, age $M=25.67$ ) at St. Petersburg State University, Russia, participated voluntarily in a single experimental session lasting approximately $30 \mathrm{~min}$. The data from two observers were excluded because their response times on test trials were too slow $(M=1464$ and $M=1871 \mathrm{~ms})$, compared with other observers $(M=$ $1064 \mathrm{~ms}$ ). Following our previous studies (Chetverikov et al., 2016, 2017b, 2017c, 2017d), the design of this study utilized within-subject comparisons with a relatively small number of trained observers (each observer was trained for at least 100 trials before the main session) performing a large number of trials. The sample size and the trial numbers were similar to those in previous studies using the same paradigm.

Method. We used a task similar to our previous studies (Chetverikov et al., 2016, 2017b). Stimuli were presented on an Acer V193 display (19" with $1280 \times 1024$ pixel resolution) using PsychoPy 1.84.2 (Peirce, 2007, 2009). Viewing distance was $\sim 60 \mathrm{~cm}$. Observers searched for two oddly oriented lines in a $6 \times 6$ grid of 36 lines subtending $16^{\circ} \times 16^{\circ}$ at the centre of a display. The length of each line was $1.41^{\circ}$. Line positions were jittered by randomly adding a value between $\pm 0.5^{\circ}$ to both vertical and horizontal coordinates.

Observers were instructed to search for two targets on each trial, with targets being the stimuli that were most different from all the others ("odd-one-out" search (Maljkovic \& Nakayama, 1994)). Targets were randomly distributed between the four quadrants of the search display with the constraint that the two targets on a given trial could not appear in the same quadrant. Observers reported the locations of the targets by pressing one of four keys (' $\mathrm{f}$ ', ' $\mathrm{g}$ ', ' $r$ ', ' $t$ ' on a standard keyboard) corresponding to the quadrants of the search display. They were informed that two targets would be presented on each trial and were encouraged to respond to each target as soon as they found it and not wait until both targets were found.

Trials were organized in intertwined prime and test 'streaks'. During prime streaks, distractors were randomly drawn from a bimodal distribution that included two uniform parts with orientations ranging from -30 to -20 and +20 to +30 relative to the overall mean. The 
135 distribution mean was the same within streak but chosen randomly between streaks. Target

136 orientations were selected randomly on each trial with the restriction that the distance between

137 target orientation and distractor mean in feature space was 60 degrees at minimum. Prime streak

138 length was set to 6-7 trials (with equal probability) because this streak length is sufficient to learn

139 bimodal distributions with relative accuracy (Chetverikov et al., 2017b).

140 Within test streaks, distractor orientations were randomly drawn from a truncated

141 Gaussian with $\mathrm{SD}=10 \mathrm{deg}$. and range $20 \mathrm{deg}$. Test streaks had one or two trials (with equal

142 probability). Different target types were used on test trials: targets were either located on a peak

143 of the previous bimodal distribution ("Peak", at $+/-25$ deg. relative to the previous distractor

144 mean), between the peaks ("Between", at 0 deg.) or outside the previous distribution range

145 ("Outside", at +/- 50 deg.). Four types of test streaks were used: 1) with two targets either on two

146 different peaks ("Peak + Peak"); 2) on a peak and in-between the peaks ("Peak + Between"); 3)

147 on a peak and outside the previous distribution range ("Peak + Outside" - where the "outside"

148 target was always $25 \mathrm{deg}$. away from the target peak, that is, either the two targets were oriented

149 at +25 and $+50 \mathrm{deg}$. or -25 and -50 relative to the previous distractors' mean); 4) between the

150 peaks and outside the range (“Outside + Between”). These four test types were presented equally

151 often (40 repetitions by participant) in random order. The distractor mean was chosen to be

152 equidistant from both test targets. The second test trial is not analyzed here as the priming effects

153 from the learning streak are not likely to be significant after the first two-target test search. Two-

154 trial test streaks were added for consistency with previous studies and in order to reduce the

155 potential effects of observers' expectations regarding streak lengths.

156 Observers participated in one session of approximately 1300 trials. Decision time was not

157 limited but participants were encouraged to respond as quickly and accurately as possible.

158 Feedback based on search time and accuracy on previous trials was shown in the upper-left

159 corner of the screen to motivate participants (see Chetverikov et al., 2016, for details on feedback

160 score calculation). The current trial number and the total number of trials were shown beneath

161 the score. If observers made an error, the word "ERROR" appeared in red letters at display centre

162 for 1 second.

163 In addition to this two-target search experiment, we also ran a single-target search study

164 (see Supplementary Experiment). The latter was used as a comparison for the single-target 
search time analyses to ensure that the introduction of a second target and specific conditions of the main experiment did not affect the pattern of results.
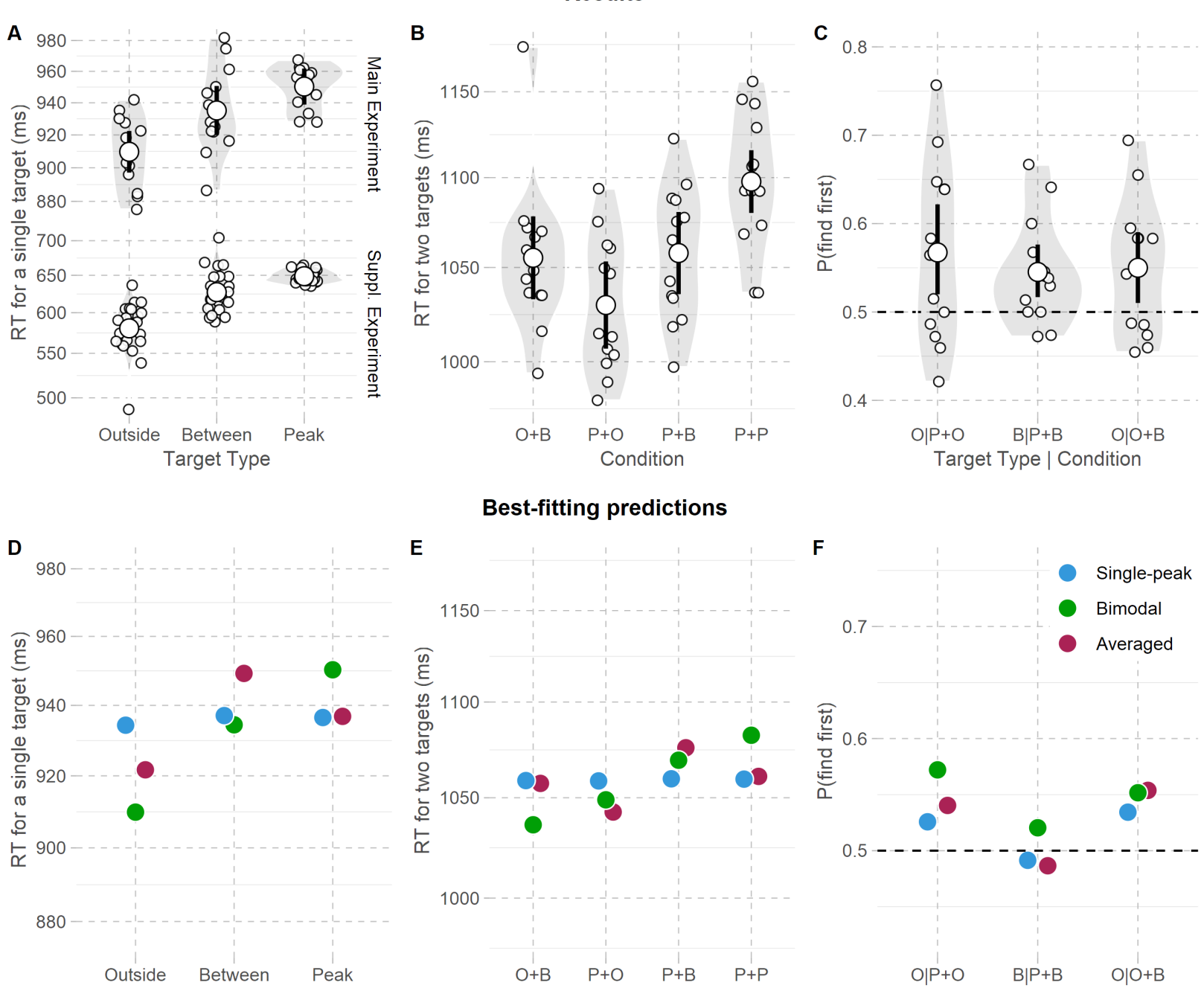

Figure 2. Experimental results and best-fitting predictions of the models (see Simulations). A: Results for different target types from the main Experiment (average search times ignoring the order in which the targets were reported) and the supplementary Experiment where observers searched for only one target on each trial. B: Results for two-target search from the main Experiment. C: Results for the order of target reporting from the main Experiment. D-F: Predictions for single-target search times, search times for two targets, and for the order in which targets would be reported in a two-target search. For A-C large dots show group means, bars show their $95 \%$ confidence intervals, smaller dots show individual observers' means, and shaded areas show distributions of individual observers' means. Abbreviations: RT - response times, $\mathrm{P}$ - target on a peak, B - target between the peaks, $\mathrm{O}$ - target outside the range of previous distractor distribution. The plus sign indicates that two targets of corresponding types are used. For the order of reporting, $\mathrm{X} \mid \mathrm{A}+\mathrm{B}$ means that target type $\mathrm{X}$ was reported first when target types $\mathrm{A}$ and $\mathrm{B}$ are combined. 
181

\section{Results}

Overall performance. On learning trials, observers found both targets in most cases $(M$ $=0.72[0.67,0.77])$, though the share of trials where only one target was reported was high $(M=$ $0.27[0.22,0.31]$; both targets were reported incorrectly on $1 \%$ of trials). On test trials, observers reported both targets correctly on $M=0.91[0.89,0.93]$ trials (accuracy was comparable to the results of single-target search in the Supplementary Experiment). The delay between the report on the first and the second target was relatively short, but longer on learning than on test trials $(M=263[198,326]$ vs. $M=176[130,233]$, respectively, $t(12.0)=4.13, p=.001)$. Similarly, the first target was reported later on learning than test trials $(M=973[854,1103]$ vs. $M=826$ $[753,904]$, respectively, $t(12.0)=5.23, p<.001)$.

The learning effects were also comparable to those from the single-target search experiment (see supplement). A linear mixed-effects regression with Helmert contrasts (comparing each trial with the average of the following trials) showed that the first trial was slower, $(B=0.11, S E=0.01, t(52.57)=9.61, p<.001)$ and less accurate $(B=-0.04, S E=0.02$, $t(13.12)=-2.62, p=.021)$ than the later trials. The follow-up trials did not differ from one another.

Test trials. Replicating previous results, search times differed depending on target type $\left(F(2,24)=8.28, p=.003, \eta^{2}{ }_{\mathrm{G}}=.02\right.$, see Figure 2A). Observers search longer for "Peak" targets compared to "Between" targets, which were in turn, found later than "Outside" targets.

Crucially, a repeated-measures ANOVA indicated that the time needed to find both targets on test trials was affected by the condition $\left(F(3,36)=6.66, p=.002, \eta^{2}{ }_{\mathrm{G}}=.02\right.$, Figure $\left.2 \mathrm{~B}\right)$.

Comparisons between conditions with the same feature difference between the targets showed that performance on "Peak + Peak" trials was slower than on "Outside + Between" trials ( $t(12.0)$ $=3.10, p=.009)$, while "Peak + Between" trials were not different from "Peak + Outside" $(t(12.0)=-1.68, p=.118)$ trials. Finally, the "Peak + Peak" condition was also slower than the "Peak + Between" condition $(t(12.0)=2.58, p=.024)$.

We then analyzed which type of target was reported first in each condition using a binomial mixed-effects regression. The results showed that targets on peaks were reported after targets between the peaks $(Z=-2.01, p=.044$, Figure $2 \mathrm{C})$ or targets outside the preceding 
210 distribution range $(Z=-2.43, p=.015)$, while the latter were reported earlier than targets

211 between the peaks $(Z=2.08, p=.037)$.

212 In sum, search with two targets on the peaks was the most difficult. A comparison of the

213 "Peak + Between" and "Peak + Outside" conditions showed only a numerical difference in total

214 RT. However, in the "Peak + Between" condition, targets on peaks were reported later than

215 targets between the peaks, whereas in the "Outside + Between" condition targets between the

216 peaks were reported later than the "Outside" targets. This shows again that targets on peaks were

217 the most unexpected for observers, followed by targets between the peaks, followed in turn by

218 "Outside" targets that led to the fastest search times.

219 Simulations. We simulated the predictions from three models (Figure 2E-F; the

220 simulation code is available at https://osf.io/rg2h8). For our main model of interest, the

221 "bimodal" model, we assumed that the probabilities of different distractors can be represented by

222 two Gaussian templates (for simplicity, we ignore the fact that the stimuli distributions might be

223 more accurately represented by non-Gaussian templates (Chetverikov, Campana, \& Kristjánsson,

224 2017a)) centered on the means of distractor distribution segments. We assumed that observers

225 utilize the knowledge they obtained about distractors and targets optimally. To find a target in a

226 localization search task, an ideal observer, would compare the probability that a given noisy

227 measurement of orientation $x$ at each location $L$ is a target versus the probability that it is a

228 distractor (Ma, Navalpakkam, Beck, van den Berg, \& Pouget, 2011; Ma, Shen, Dziugaite, \& van

229 den Berg, 2015):

$$
p(L \mid x) \propto p(L) \frac{p(x \mid T)}{p(x \mid D)}=p(L) \frac{p\left(x \mid s_{L}\right) p\left(s_{L} \mid T\right) d s_{L}}{p\left(x \mid s_{L}\right) p\left(s_{L} \mid D\right) d s_{L}}
$$

231 where $s_{L}$ is a true stimulus value at this location, $p(L)$ is the probability that a target is presented 232 at this location, $T$ and $D$ are the parameters of target and distractor distributions, respectively. In

233 our simulations, we assumed that internal representations of target and distractor distributions are

234 independent and response times are inversely proportional to the amount of evidence $p(L \mid x)$.

235 Given that all locations in our experiment were equiprobable, that is, $p(L)$ is the same for all

236 locations, response times will, on average, be proportional to the probabilities of the test target $\theta_{T}$ 237 under given distractor template parameters:

$$
R T \propto p\left(\theta_{T} \mid D\right)
$$


The width of the Gaussian templates was estimated by fitting the model to single-target response time data. To increase the robustness of the estimates, we used an approach similar to bootstrap aggregating ("bagging"), often employed in machine learning (Breiman, 1996). For each model we obtained 500 bootstrapped samples grouped by participant (that is, on each iteration, sampling with replacement was done for each subject and then the samples were combined). We then estimated the template widths for each sample by fitting response times as a linear function of the stimuli probability. For a "bimodal" model:

$$
R T_{1 T}=a+b\left(\frac{1}{2} p\left(\theta_{T} \mid \mu_{1}, \sigma\right)+\frac{1}{2} p\left(\theta_{T} \mid \mu_{2}, \sigma\right)\right)
$$

where $\mu_{1}=25$ and $\mu_{2}=-25$, the means of bimodal distractor distribution peaks, and $a$ and $b$ are the scaling parameters necessary to translate the probabilities into response times. The template widths obtained for each sample were then averaged to get the resulting estimates.

250 Estimated template widths were similar for the experiment reported here (18 deg.) and the 251 supplemental experiment (21 deg.).

For the "single-peak" model, we assumed that only one of the two peaks was encoded 254 equidistant to the overall distractor mean:

$$
R T_{1 T}=a+b\left(p\left(\theta_{T} \mid \mu_{1}, \sigma\right)\right)
$$

The estimated template widths were 27 and $22 \mathrm{deg}$. for the main and the supplemental experiment.

Finally, the "averaged" model was based on the idea that observers might use a single set of summary statistics to represent the stimuli. Accordingly, we assumed that observers use a single Gaussian template centered at the mean of the overall bimodal distribution: main experiment the estimated width was $114 \mathrm{deg}$., while for the supplemental experiment it was

264140 deg. (i.e., almost flat template), already suggesting that this model provides a poor fit to the 265 experimental data.

We then used the estimated template widths to obtain the predictions of the three models

267 for the search times for different target types (Figure 2D), total search time for two targets in 268 different conditions (Figure 2E), and for the order in which the targets should be reported (Figure 
2F). For single-target search the equations were the same as when we estimated the template widths, however, we used the data averaged by target type for each subject to reduce the effect of trial-by-trial variability. Two-target search times were assumed to be proportional to a sum of two search times predicted in the same way as for a single target:

$$
R T_{2 T}=a+b\left(p\left(\theta_{T 1} \mid D\right)+p\left(\theta_{T 2} \mid D\right)\right)
$$

where $D$ reflects the distractor distribution parameters for a given model, that is, the template mean(s) and its estimated width(s).

Finally, we assumed that all other things being equal, the order in which the targets are reported would depend on the ratio of the probabilities of observing the test targets under the given distractors template:

$$
P(\text { find } T 2 \text { first })=0.5+k \log \left(\frac{p\left(\theta_{T 1} \mid D\right)}{p\left(\theta_{T 2} \mid D\right)}\right)
$$

with $k$ as a scaling constant. The ratio was transformed to logarithm to allow for both positive and negative values.

Figures $2 \mathrm{D}$ and $2 \mathrm{E}$ show that the bimodal model provided more accurate predictions for response times than the other models. For single-target response times, it accurately predicted that targets on peaks would be the hardest to find and targets between the peaks would be harder to find than targets outside the range of previously learned distractors. In contrast, the averaged model $(\triangle \mathrm{BIC}=5.94 ;$ here and later $\triangle \mathrm{BIC}$ refers to the difference in Bayesian Information Criterion compared to the bimodal model, positive values meaning that the bimodal model has better fit) suggested that the targets in-between the peaks would be hardest to find, while the single-peak model $(\triangle \mathrm{BIC}=12.47)$ predicted relatively similar response times for between targets and targets on peaks. For two-target RTs, the bimodal model failed to predict slower search for the "outside + between" condition compared to the "peak + outside" condition. Note, however, that this difference was also not significant in our results. Speculatively, it might be a result of a higher similarity between the targets in the latter than in the former. Nevertheless, the predictions of the bimodal model were still better than of the averaged $(\Delta \mathrm{BIC}=8.04)$ or the single-peak model $(\triangle \mathrm{BIC}=6.59)$.

Crucially, the bimodal, single-peak, and averaged models gave qualitatively different predictions for the order in which the targets would be found. For both the single-peak and averaged model, the probability of first reporting targets between the peaks when combined with 
299 targets on peaks was below 0.5 (Figure 2C). As outlined in the introduction, when observers 300 encode only one peak, on $50 \%$ of the trials, the "peak" target on test trials should be on this peak 301 while in the other half of the trials it will be on the non-encoded peak. Depending on the width of 302 the template, the average ratio of the probabilities for a target would vary: with very large or very 303 small template widths, it will be close to 0.5 because targets between the peaks and at the non304 encoded peaks will be equally probable, and with intermediate template widths it will be below 3050.5 (note that this conclusion is not limited to the specific equation we used for determining the 306 probability of finding one target before another; in fact, it could be shown that this is the case for 307 any monotonic function describing the transformation of a ratio of probabilities of observing the 308 target under a given Gaussian distractor template into average probability of a given reporting 309 order). For the averaged model the target between the peaks should always be reported later than 310 targets on the peaks. In contrast, for the bimodal model that accurately encodes the probabilities 311 of distractors, the target between the peaks should be reported before the target at the peak. 312 Accordingly, the bimodal model describes the results better than the single-target $(\triangle \mathrm{BIC}=$ $31313.11)$ or the averaged model $(\triangle \mathrm{BIC}=6.86)$. 


\section{Discussion}

316 Can observers develop an accurate internal model for the probabilities of to-be-ignored items in a

317 visual search task? We assessed the content of templates guiding visual search in the orientation

318 domain, by measuring slowing for targets drawn from a preceding distractor orientation

319 distribution. The distribution was bimodal and the searches used to probe the representations

320 involved two simultaneous targets within a trial. Response times were slower when the targets

321 corresponded to the two modes ("peaks") of previous distractor distributions than when one

322 target was from one of the modes and another from between them, while the latter combination

323 of targets resulted in slower search than when one of the targets was outside the previous

324 distractor range. Furthermore, the order in which the targets were reported on a test trial followed

325 the distractor probabilities observed during prime trials. Targets outside the previous distractor

326 range were reported earlier than the ones between the modes, while the latter were reported

327 before the targets at the modes of previous distractor distribution. The search times and the order

328 in which targets are reported allowed us to assess the internal model used by observers.

329 We simulated the predictions of a bimodal, single-template, and averaged template

330 models. The first model accurately reflects the actual distribution of distractor features, while the

331 other two oversimplify it in different ways. We found that the bimodal model predicts the

332 response times pattern for different target types and different conditions far better than the other

333 models. Moreover, only the bimodal model could accurately predict the order in which the

334 targets were reported. Both the single-template and the averaged-template model predicted that

335 the target between the peaks should on average be reported after the targets at the peaks, while

336 the reverse was accurately predicted by the bimodal model. The target between the peaks in the

337 "Peak + Between" condition was on average reported before the target at one of the peaks. This

338 shows that observers simultaneously represent both modes of distractor distributions. Their

339 representations approximate the physical stimuli, and they fill in the gaps in probability space as

340 demonstrated by slower responses when one of the targets was between the peaks compared to

341 when it was outside the previous distractor range, or on the peaks.

342 Notably, all three models can be considered probabilistic in a sense that they do provide

343 observers with a measure of probability that a certain feature belongs to a distractor class. The

344 difference is in the degree of simplification. The bimodal model reflects the probability 
345 distribution accurately (with the assumption of Gaussian approximation). The two other models taken into consideration, however, diverge from an accurate representation in different ways: the "averaged" assumes the use of overall summary statistics, while the "single-peak" assumes the encoding of only one part of the distribution (which could be caused, for example, by biased sampling). Furthermore, every heuristic or decision rule can be cast in terms of probabilities (e.g., a delta function that assigns probability of 1 for one part of feature space and 0 for the rest). Here we show that the representations used by observers mirror the probability distribution of the 352 stimuli.

Unlike previous studies assessing how distracting information is stored in visual working

354 memory (Arita et al., 2012; Won \& Geng, 2018), the distractors in our studies were

355 heterogeneous and were generated randomly based on a bimodal probability distribution.

356 Nevertheless, observers were able to integrate the information about distractors into an

357 approximate bimodal representation. Speculatively, this demonstrates that using homogeneous

358 distractors may be an artificial limitation, perhaps brought on by earlier technical restrictions on

359 experimental stimuli in pre-modern computer era. In the real world, distracting information is

360 rarely homogeneous, so it may not be particularly surprising that humans are able to form accurate templates representing probability distributions.

Following seminal accounts of priming of pop-out effects (Maljkovic \& Nakayama,

363 1994), we argue that the representations of distractor distributions are kept in visual working 364 memory, rather than long-term memory. Woodman et al (Woodman et al., 2013) have 365 demonstrated that the representation of a single attended target is transferred from VWM to long366 term memory in 5 to 7 trials. In contrast, we have previously shown that for simple distractor 367 distributions (such as Gaussian or uniform) one or two trials are enough for observers to develop 368 a probabilistic representation of distractors (Chetverikov et al., 2017b). Representations of more 369 complex distractor distributions take more time (or trials) to develop, but they also progressively 370 change with more repetitions: after one or two trials, bimodal distributions are represented as 371 unimodal, and are only later transformed into bimodal ones. This indicates that more time (trials) 372 is required for sharpening the representation, not for the transfer to long-term memory.

373 A question of how the probabilistic templates for rejection are stored also taps into a 374 more general question, regarding how working memory templates are stored. Recently, 375 Christophel, Iamschinina and colleagues (2018) demonstrated that while attended stimuli in 
376 visual working memory are represented both in parietal and frontal cortex in addition to visual

377 cortex, the latter is not involved in representations of unattended stimuli. It is possible that

378 rejection templates similarly do not involve early visual areas. However, unlike simple

379 unattended items, templates for rejection are actively used by observers to guide attention. As

380 such, their representation might require a level of precision only achievable with the recruitment

381 of sensory areas.

$382 \quad$ How specific are distractor templates? Won and Geng (Won \& Geng, 2018) suggested

383 that distractor templates might be more broadly tuned than target templates. This would allow

384 easy generalization of suppression to similar distractors, while for targets such generalization

385 might be harmful as it would lead to an increased number of false alarms. However, the exact

386 costs of generalization for both target and distractor templates depend on the environment.

387 Specific templates are necessary when a target is similar to distractors, but generalization is

388 helpful otherwise. This has indeed been observed by Geng, DiQuattro, and Helm (Geng,

389 DiQuattro, \& Helm, 2017): when a target is similar to distractors, its template is sharpened and

390 shifted away from distractors. Moreover, in the real environment we rarely know how exactly the

391 target or distractors would look under a given illumination and point of view, making some

392 degree of generalization essential for efficient search. In contrast, a typical visual search study

393 would require a very narrow distribution of target features, making a narrow template useful. Our

394 results suggest that distractor templates are specific enough to account for bimodality in the

395 distractor distribution. It remains to be studied whether targets or distractors templates are more

396 specific when their physical distributions are equally shaped.

397 In contrast to our previous studies (Chetverikov et al., 2016, 2017b, 2017d, 2017c;

398 Hansmann-Roth, Chetverikov, \& Kristjánsson, 2019), here, we "probed” the distractor

399 representation only at three different points in the feature space. By using targets with a range of

400 features that covered the full feature space, our previous research showed that observers encode

401 the probability distribution of distractors. Here we extend these findings by showing that

402 observers learn the distribution of distractors following a single learning streak. This

403 demonstrates that the previously obtained results are not an artefact of aggregation over multiple

404 trials but rather a true reflection of the templates' content.

405 Our results agree with previous findings on probabilistic concept learning. Briscoe and

406 Feldman (2011) found that when observers have to form a decision rule based on a multimodal 
407 probability distribution, they could do this, although performance became worse with increased 408 mode number. We did not explicitly ask our observers to categorize the stimuli (as distractors 409 and targets), but it is conceivable that they might do so if asked.

410 We should note that one might interpret our results as simply demonstrating that humans 411 are capable of learning a nonlinear classification rule/decision boundary over a disjoint set in 412 feature space, and can use this to guide visual search. But we think that this alternative proposal 413 is unlikely to hold water because for a simple classifier in this task, learning is not necessary.

414 There is enough information on each trial to easily tell the target from distractors. Moreover, to 415 include learning in the algorithm, learning of the target would suffice, as the target distribution is 416 constant within the learning streak. The fact that our observers struggle with this shows that they

417 do more than strictly necessary. Second, and perhaps more importantly, we showed in our 418 previous work that observers learn the correct probabilities of the distractor features on average 419 rather than learning a simple decision rule (Chetverikov et al., 2016, 2017b, 2017d, 2017c). A 420 decision rule model cannot explain why the response time curves reflect distractor probability 421 both within and outside the distractor distribution range. By using double-target search we 422 further demonstrate that these results cannot be explained by a combination of different decision 423 rules applied on different test trials.

\section{Conclusions}

425 We found that rejection templates are probabilistic, similarly to items in visual working 426 memory that receive attention (Ma et al., 2014). However, our study also shows that templates 427 for rejection do not need to be simple bell-shaped curves, as it is typically modelled in working 428 memory studies. In contrast, they are dynamically adapted to task requirements, reflecting the 429 probabilistic nature of the input. Whether such flexibility also characterizes templates for 430 attended items remains to be seen. However, our results clearly demonstrate that probabilistic 431 computations start in the brain even before something is perceived, to determine what should be 432 prioritized in perception. 


\section{Acknowledgments}

434 Supported by a grant from the Icelandic Research Fund (IRF \#173947-052). AC was supported 435 by the Radboud Excellence Initiative. We are grateful to Alena Begler for help with data 436 collection.

\section{Supplementary materials}

438 The data from the experiments reported in this paper and scripts for simulations and analyses are 439 available at https://osf.io/rg2h8. The data include trial-by-trial on presented stimuli and 440 observers' responses. The analyses scripts in $\mathrm{R}$ provide the full pipeline, including the data 441 preprocessing.

443 All authors participated equally in conceiving and planning the experiments. AC wrote the 444 experimental scripts, oversaw the data collection, analyzed the results, and wrote the initial 445 version of the manuscript. GC and $\mathrm{AK}$ took part in data analyses and interpretation and revised 446 the manuscript. 


\section{References}

449

Alvarez, G. A. (2011). Representing multiple objects as an ensemble enhances visual cognition. Trends in Cognitive Sciences, 15(3), 122-131. https://doi.org/10.1016/j.tics.2011.01.003

Arita, J. T., Carlisle, N. B., \& Woodman, G. F. (2012). Templates for rejection: Configuring attention to ignore task-irrelevant features. Journal of Experimental Psychology: Human Perception and Performance, 38(3), 580-584. https://doi.org/10.1037/a0027885

Bays, P. M. (2015). Spikes not slots: Noise in neural populations limits working memory. Trends in Cognitive Sciences, 19(8), 431-438. https://doi.org/10.1016/j.tics.2015.06.004

Bejjanki, V. R., Beck, J. M., Lu, Z.-L., \& Pouget, A. (2011). Perceptual learning as improved probabilistic inference in early sensory areas. Nature Neuroscience, 14(5), 642-648. https://doi.org/10.1038/nn.2796

Breiman, L. (1996). Bagging predictors. Machine Learning, 24(2), 123-140. https://doi.org/10.1007/BF00058655

Briscoe, E., \& Feldman, J. (2011). Conceptual complexity and the bias/variance tradeoff. Cognition, 118(1), 2-16. https://doi.org/10.1016/j.cognition.2010.10.004

Bundesen, C. (1990). A theory of visual attention. Psychol Rev, 97(4), 523-547. https://doi.org/10.1037/0033-295X.97.4.523

Chetverikov, A., Campana, G., \& Kristjánsson, Á. (2016). Building ensemble representations: How the shape of preceding distractor distributions affects visual search. Cognition, 153, 196-210. https://doi.org/10.1016/j.cognition.2016.04.018

Chetverikov, A., Campana, G., \& Kristjánsson, Á. (2017a). Learning features in a complex and changing environment: A distribution-based framework for visual attention and vision in general. In Progress in Brain Research (Vol. 236, pp. 97-120). Elsevier. https://doi.org/https://doi.org/10.1016/bs.pbr.2017.07.001

Chetverikov, A., Campana, G., \& Kristjánsson, Á. (2017b). Rapid learning of visual ensembles. Journal of Vision, 17(21), 1-15. https://doi.org/10.1167/17.2.21

Chetverikov, A., Campana, G., \& Kristjánsson, Á. (2017c). Representing Color Ensembles. Psychological Science, 28(10), 1-8. https://doi.org/10.1177/0956797617713787

Chetverikov, A., Campana, G., \& Kristjánsson, Á. (2017d). Set size manipulations reveal the boundary conditions of distractor distribution learning. Vision Research, 140(November), 
144-156. https://doi.org/10.1016/j.visres.2017.08.003

Chetverikov, A., Hansmann-Roth, S., Tanrikulu, Ö. D., \& Kristjansson, Á. (2019). Feature Distribution Learning (FDL): A New Method for Studying Visual Ensembles Perception with Priming of Attention Shifts. In Neuromethods (pp. 1-21). Springer. https://doi.org/10.1007/7657_2019_20

Christophel, T. B., Iamshchinina, P., Yan, C., Allefeld, C., \& Haynes, J. D. (2018). Cortical specialization for attended versus unattended working memory. Nature Neuroscience, 21(4), 494-496. https://doi.org/10.1038/s41593-018-0094-4

Feldman, J. (2014). Probabilistic models of perceptual features. In J. Wagemans (Ed.), Oxford Handbook of Perceptual Organization (pp. 933-947). https://doi.org/10.1093/oxfordhb/9780199686858.013.049

Geng, J. J., DiQuattro, N. E., \& Helm, J. (2017). Distractor probability changes the shape of the attentional template. Journal of Experimental Psychology: Human Perception and Performance, 43(12), 1993-2007. https://doi.org/10.1037/xhp0000430

Girshick, A. R., Landy, M. S., \& Simoncelli, E. P. (2011). Cardinal rules: visual orientation perception reflects knowledge of environmental statistics. Nature Neuroscience, 14(7), 926932. https://doi.org/10.1038/nn.2831

Grubert, A., \& Eimer, M. (2013). Qualitative differences in the guidance of attention during single-color and multiple-color visual search: Behavioral and electrophysiological evidence. Journal of Experimental Psychology: Human Perception and Performance, 39(5), 14331442. https://doi.org/10.1037/a0031046

Hansmann-Roth, S., Chetverikov, A., \& Kristjánsson, Á. (2019). Representing color and orientation ensembles: Can observers learn multiple feature distributions? Journal of Vision, 19(9), 1-17. https://doi.org/10.1167/19.9.2

Kersten, D., Mamassian, P., \& Yuille, A. (2004). Object Perception as Bayesian Inference. Annual Review of Psychology, 55(1), 271-304. https://doi.org/10.1146/annurev.psych.55.090902.142005

Kristjánsson, Á., \& Driver, J. (2008). Priming in visual search: separating the effects of target repetition, distractor repetition and role-reversal. Vision Research, 48(10), 1217-1232. https://doi.org/10.1016/j.visres.2008.02.007

Lamy, D. F., Antebi, C., Aviani, N., \& Carmel, T. (2008). Priming of Pop-out provides reliable 
measures of target activation and distractor inhibition in selective attention. Vision Research, 48(1), 30-41. https://doi.org/10.1016/j.visres.2007.10.009

Ma, W. J. (2012). Organizing probabilistic models of perception. Trends in Cognitive Sciences, 16(10), 511-518. https://doi.org/10.1016/j.tics.2012.08.010

Ma, W. J., Husain, M., \& Bays, P. M. (2014). Changing concepts of working memory. Nature Neuroscience, 17(3), 347-356. https://doi.org/10.1038/nn.3655

Ma, W. J., Navalpakkam, V., Beck, J. M., van den Berg, R., \& Pouget, A. (2011). Behavior and neural basis of near-optimal visual search. Nature Neuroscience, 14(6), 783-790. https://doi.org/10.1038/nn.2814

Ma, W. J., Shen, S., Dziugaite, G., \& van den Berg, R. (2015). Requiem for the max rule? Vision Research, 116, 179-193. https://doi.org/10.1016/j.visres.2014.12.019

Maljkovic, V., \& Nakayama, K. (1994). Priming of pop-out: I. Role of features. Memory \& Cognition, 22(6), 657-672. Retrieved from http://www.ncbi.nlm.nih.gov/pubmed/7808275

Oberauer, K. (2002). Access to Information in Working Memory: Exploring the Focus of Attention. Journal of Experimental Psychology: Learning Memory and Cognition, 28(3), 411-421. https://doi.org/10.1037//0278-7393.28.3.411

Olivers, C. N. L., Peters, J., Houtkamp, R., \& Roelfsema, P. R. (2011). Different states in visual working memory: When it guides attention and when it does not. Trends in Cognitive Sciences, 15(7), 327-334. https://doi.org/10.1016/j.tics.2011.05.004

Peirce, J. W. (2007). PsychoPy--Psychophysics software in Python. Journal of Neuroscience Methods, 162(1-2), 8-13. https://doi.org/10.1016/j.jneumeth.2006.11.017

Peirce, J. W. (2009). Generating stimuli for neuroscience using PsychoPy. Frontiers in Neuroinformatics, 2. https://doi.org/10.3389/neuro.11.010.2008

Rao, R. P., Olshausen, B. A., \& Lewicki, M. S. (2002). Probabilistic models of the brain: Perception and neural function. MIT Press.

van Moorselaar, D., Theeuwes, J., \& Olivers, C. N. L. (2014). In competition for the attentional template: Can multiple items within visual working memory guide attention? Journal of Experimental Psychology: Human Perception and Performance, 40(4), 1450-1464. https://doi.org/10.1037/a0036229

Vickery, T. J., King, L.-W., \& Jiang, Y. (2005). Setting up the target template in visual search. Journal of Vision, 5(1), 8. https://doi.org/10.1167/5.1.8 
540 Wang, D., Kristjánsson, Á., \& Nakayama, K. (2005). Efficient visual search without top-down or 541 bottom-up guidance. Perception \& Psychophysics, 67(2), 239-253.

542 https://doi.org/10.3758/BF03206488

543 Won, B.-Y. Y., \& Geng, J. J. (2018). Learned suppression for multiple distractors in visual

544 search. Journal of Experimental Psychology: Human Perception and Performance, 44(7),

545 1128-1141. https://doi.org/10.1037/xhp0000521

546 Woodman, G. F., Carlisle, N. B., \& Reinhart, R. M. G. (2013). Where do we store the memory

547 representations that guide attention? Journal of Vision, 13(3), 1-1.

$548 \quad$ https://doi.org/10.1167/13.3.1

549

550 


\section{Supplemental experiment}

\section{Method}

Participants. Fifteen observers (ten female, age $M=25.47$ ) at St. Petersburg State University, Russia, took part in a single experimental session lasting approximately $30 \mathrm{~min}$. Two of them were excluded because their response times were very high compared with the other observers (1356 and $1393 \mathrm{~ms}$ for excluded observers vs. $761 \mathrm{~ms}$ for the remaining sample). One more was excluded because of low accuracy $(M=0.71$ vs. 0.83 for the remaining sample). The study was approved by the ethics committee of St. Petersburg State University.

Procedure. We used a task similar to our previous studies (Chetverikov et al., 2016, 2017b). Stimuli were presented on an Acer V193 display (19" with $1280 \times 1024$ pixel resolution) using PsychoPy 1.84.2 (Peirce, 2007, 2009). Viewing distance was $\sim 60 \mathrm{~cm}$. Observers searched for an oddly oriented line in a $6 \times 6$ grid of 36 lines subtending $16^{\circ} \times 16^{\circ}$ at the centre of a display. The length of each line was $1.41^{\circ}$. Line positions were jittered by randomly adding a value between $\pm 0.5^{\circ}$ to both vertical and horizontal coordinates. Observers indicated whether the target line was in the upper or the lower half of the screen by pressing the ' $\mathrm{i}$ ' or ' $\mathrm{j}$ ' keys on a standard keyboard. Trials were organized in intertwined prime and test 'streaks'. During prime streaks, distractors were randomly drawn from a bimodal distribution that included two uniform parts with orientations ranging from -40 to -20 and +20 to +40 relative to the overall mean. The distribution mean was the same within streak but chosen randomly between streaks. Target orientation was selected randomly on each trial with the restriction that the distance between target orientation and distractor mean in feature space was 60 degrees at minimum. Based on the results of previous studies, prime streak length was set to 6-7 trials because this streak length is sufficient to encode bimodal distributions with relative accuracy (Chetverikov et al., 2017b).

Within test streaks, distractor orientations were randomly drawn from a truncated Gaussian with SD = $10 \mathrm{deg}$. and range $20 \mathrm{deg}$. Each test streak had two trials and the targets on these trials (target type) were either located on the "peaks" of the previous bimodal distribution (within the +/- 25 to $35 \mathrm{deg}$. range relative to the previous distractors' mean), in-between the peaks (within 0 to $+/-5$ deg. range) or outside the previous distribution range (within $+/-55$ to 90 deg. range). Three types of test streaks were used with targets on the first and the second test trial either on two different peaks, on a peak and in-between the peaks, or on a peak and outside the 
581 582

previous distribution range. These three conditions were presented equally often in random order. The order of targets within the test trials for each condition was counterbalanced. The distractor mean was chosen randomly with a distance to the target of no less than $60 \mathrm{deg}$. (as on prime trials).

Observers participated in one session of approximately 1244 trials divided into 288 prime and test streaks. Decision time was not limited but participants were encouraged to respond as quickly and accurately as possible. Feedback based on search time and accuracy on previous trials was presented after each trial was shown in the upper-left corner of the screen to motivate participants. The current trial number and the total number of trials were shown beneath the score. If observers made an error, the word "ERROR" appeared in red letters at display centre for 1 second.

\section{Results}

Overall performance. Participants were slower $(M=738[683,794]$ vs. $M=615[589$, 643], $t(11.0)=5.67, p<.001, d=1.64)$ and less accurate $(M=0.78[0.75,0.82]$ vs. $M=0.96$ $[0.95,0.97], t(11.0)=-10.29, p<.001, d=2.97)$ on learning trials than test trials, due to the fact that learning trials had a broader distribution. Response times decreased while accuracy increased during learning trials: A linear mixed-effects regression indicated that the first trials in each learning sequence were slower, $(B=0.08, S E=0.02, t(11.47)=4.52, p<.001)$ and less accurate $(B=-0.04, S E=0.01, t(12.52)=-4.50, p<.001)$ than the later trials.

Test trials. On test trials, observers' performance depended on both target type and condition. Replicating the results of Chetverikov et al. (2017b), on the first trial in a test sequence observers responded more slowly when the target was at one of the peaks of the preceding distractor distribution than when it was in-between the peaks $(t(11.0)=3.94, p=.002$, $d=1.14$ ), while responses for the in-between the peaks targets were slower than when they were outside the range of the previous distribution $(t(11.0)=3.96, p=.002, d=1.14)$. 